\title{
Testing Stress and Dyadic Coping Processes in Chinese Couples
}

\author{
Feng Xu \\ University of Zurich and \\ Guangdong Construction Polytechnic
}

\author{
Fridtjof W. Nussbeck \\ University of Bielefeld
}

\author{
Peter Hilpert \\ University of Washington
}

\author{
Guy Bodenmann \\ University of Zurich
}

\begin{abstract}
Previous empirical studies show that stress occurring outside of the relationship (referred to as external stress) can spill over into couples' relationships, influencing dyads' behaviors and their relationship satisfaction. There is substantial evidence that the association between external stress and relationship satisfaction is explained by 2 mediators: internal stress and dyadic coping. However, the mediation processes of internal stress and dyadic coping have solely been examined in Western couples. Accordingly, the current study aimed to test these processes in a sample of 474 Chinese couples $(N=948)$ using the actor-partner interdependence mediation model. Results reveal that internal stress and dyadic coping can fully mediate the association between external stress and relationship satisfaction in Chinese couples for men and women in a similar way. These findings underscore the importance of moving beyond examining stress and dyadic coping processes among couples in Eastern cultures.
\end{abstract}

Keywords: Chinese couples, dyadic coping, external stress, relationship satisfaction, internal stress

Stress originating outside of couples' relationships (external stress) can spill over into their relationships (Repetti, 1989) and affect their levels of relationship satisfaction (Buck \& Neff, 2012; Randall \& Bodenmann, 2009). The way in which external stress affects their relationship satisfaction can be explained by two mediators: internal

Feng Xu, Department of Psychology, University of Zurich and Department of Student Affairs, Guangdong Construction Polytechnic; Peter Hilpert, Department of Psychiatry and Behavioral Sciences, University of Washington; Fridtjof W. Nussbeck, Department of Psychology, University of Bielefeld; Guy Bodenmann, Department of Psychology, University of Zurich.

Our manuscript has been presented at the 2015 IARR conference as a poster session but has not been published elsewhere. In addition, data was utilized to validate the Dyadic Coping Inventory, which was used in the current study and the validation study has been published in Psychological Assessment (Xu, Hilpert, Randall, Li, \& Bodenmann, 2016). This research was supported by China Scholarship Council (Grant 201308440256).

Correspondence concerning this article should be addressed to Feng Xu, Department of Psychology, University of Zurich, Binzmuehlestrasse, 14/23, 8050 Zurich, Switzerland. E-mail: xufenggz@gmail.com stress and dyadic coping (e.g., Bodenmann, Ledermann, \& Bradbury, 2007; Hilpert, Kuhn, Anderegg, \& Bodenmann, 2015). Thus far, researchers have tested stress and dyadic coping processes mainly in Western couples. Less is known about whether these mediation effects are also functional for couples living in Eastern cultures (e.g., Chinese), for whom familial interdependence is extremely emphasized (Chen \& Li, 2007). Moreover, studies show that men and women have different behavioral patterns in stress and coping processes (Bodenmann et al., 2015) and these patterns should thus be further explored in Chinese couples. Accordingly, the goal of the study was to explore stress and dyadic coping processes in a sample of Chinese couples that would expand the knowledge for researchers and practitioners in assisting couples with diverse cultural backgrounds in coping with stress.

\section{The Theoretical Model Explaining Stress and Dyadic Coping Processes}

Couples' stress and coping processes have long been an important research topic for family researchers in Western countries. Based on the interdependent and reciprocal effect of couples' 
susceptibility to stressors, the Systemic Transactional Model (STM; Bodenmann, 2005) was developed to explain couples' stress and coping processes. The STM is a conceptual model that suggests one partner's external stress can spill over into the relationship and affect their stress reactivity as well as their partner's corresponding coping behaviors (Hilpert et al., 2015; Wunderer \& Schneewind, 2008), which subsequently affects relationship outcomes (e.g., relationship satisfaction; Buck \& Neff, 2012; Herzberg, 2013). In interpreting the effects of stress and coping processes on relationship functioning, the STM highlights two mediators: internal stress and dyadic coping. Internal stress refers to perceived differences between spouses in relation to life needs, goals, values, and personality traits. Internal stress can further cause arguments and conflicts within the relationship. The role of internal stress in the mediation process is as follows: external stress exacerbates couples' personal drawbacks (e.g., hostility, intolerance) and conflict interactions (e.g., negative communication, negligence of spouses' emotions), which decreases the level of intimacy and increases dissatisfaction between partners (Ledermann, Bodenmann, Rudaz, \& Bradbury, 2010; Bodenmann et al., 2007). Furthermore, several empirical studies suggest that internal stress is a robust mediator between external stress and relationship satisfaction (Hilpert et al., 2015; Bodenmann et al., 2007; Ledermann et al., 2010).

Further, stress spillover effects can activate couples' dyadic coping behaviors. As an extension of individual coping efforts, dyadic coping is defined as a systemic and interdependent process between one stressed partner and his or her spouse who can potentially provide support (Bodenmann, 2005; Revenson \& Lepore, 2012). The STM argues that dyadic coping is a coping resource that is beneficial for couples by helping the stressed person to alleviate stress reactivity and increase intimacy and relationship satisfaction (Bodenmann, 2005). Utilizing dyadic coping resources, couples are interdependent to cope with external stress that might impair relationship functioning, which can protect them from activating internal stress. Several lines of research reveal that dyadic coping is positively associated with relationship satisfaction (see a meta-analysis, Falconier, Jackson, Hilpert, \& Bodenmann, 2015) and can mediate the associ- ation between external stress and relationship satisfaction in Western couples (Donato et al., 2015; Hilpert, Bodenmann, Nussbeck, \& Bradbury, 2013; Hilpert et al., 2015; Wunderer \& Schneewind, 2008). Nevertheless, less is known about how these mediation processes function beyond Western couples.

\section{A Cultural Perspective of Stress and Coping Processes in Chinese Couples}

Couples in China are affected by collectivism, Confucianism, and contemporary values (Shek, 2006; Quek, Knudson-Martin, Rue, \& Alabiso, 2010; Xu \& Hiew, 2016).Collectivistic culture purports that group goals are valued above personal goals (Oyserman, Coon, \& Kemmelmeier, 2002), and maintaining harmonious relationship with others is crucial (Marshall, 2008), which is especially important for married couples (Ebrey, 2006; Shek, 2006; Xu \& Hiew, 2016). Confucianism prescribes distinct gender roles for couples: husbands are breadwinners and should be seen as the head in the family whereas wives are responsible for the household and parenting (Ebrey, 2006; Zuo, 2003). However, since the Chinese government has launched up the open-up policy to foreign countries for several decades, the Chinese population is in a transition to more contemporary values as a consequence of economic advancement and the consistent exposure to Western values (Xu \& Hiew, 2016). The contemporary value highlights an egalitarian gender role for couples (Quek et al., 2010). Notably, the value of the equal gender role becomes more and more accepted among Chinese couples as Chinese men and women have equal education and job opportunities (Shek, 2006; Xu \& Hiew, 2016). For instance, several studies show that a majority of Chinese couples are dual earners (around 85\%; Ling \& Powell, 2001; Zhang, Foley, \& Yang, 2013). This transition might also affect marital expectations and behavioral exchanges in Chinese couples.

Understanding cultural and contemporary influences on Chinese couples renders us to make predictions about their stress and coping processes. Based on the traditional values, we can predict distinct gender differences in stress and coping processes. As husbands traditionally have a higher family status, they can enact more conflict behaviors and expect more support from their 
wives (e.g., Chen \& Li, 2007). However, nowadays, the 4-2-1 pattern in the Chinese family is a universal phenomenon (couples' parents-two members of the couple-one child, Hesketh, Lu, \& Xing, 2005). As a result, the demands and expectations on dual-earner couples with younger children is so high that family responsibilities are more and more equally shared between partners (Xu \& Hiew, 2016). Thus, in terms of the transition from traditional cultures to modern values we can expect to hardly find gender differences in young couples who are born after the 1980s (Shu \& Zhu, 2009). It indicates that partners may be more vulnerable to daily life stressors and therefore expect and exchange similar coping behaviors.

\section{Current Study}

In order to examine how external stress can spill over into relationships, we mainly focus on couples who are supposed to experience higher levels of stress-dual-earner couples with younger children. We hypothesized that internal stress would significantly mediate the association between external stress and relationship satisfaction (Hypothesis 1a [H1a]) and predicted that the negative effect of own stress on own relationship satisfaction was equally mediated by internal stress for men and women (H1b). Moreover, we hypothesized that dyadic coping would significantly mediate the association between external stress and relationship satisfaction (H2a); we further expected to find the same mediation mechanism of dyadic coping for men and women ( $\mathrm{H} 2 \mathrm{~b})$.

\section{Method}

\section{Participants and Procedure}

Couples were recruited in three metropolitan cities (Beijing, Shanghai, and Guangzhou) and several southern and northern provinces (e.g., Guangdong, Jiangsu, Shanxi, Jiangxi, and Sichuan) by distributing flyers at parents' meetings in schools, and to administrative managers of different enterprises (civil institutions, companies, schools, and hospitals). Participants had to meet the following criteria in order to be able to participate: (a) married and living with a partner for at least 1 year; (b) over 22 years of age for males and over 18 years of age for females (legal ages to marry in China). Interested couples who met the inclusion criteria received separate letters including information about the study, participation agreement, the questionnaires, and two preaddressed and stamped envelopes. Couples were instructed to sign the participation agreement, fill out their questionnaires separately and send the papers back in separate envelopes. Participants were notified that their data would be kept anonymous and confidential for scientific purposes. Research assistants distributed 1,000 flyers at parents' meetings in schools and to administrative managers of different enterprises. Overall, $600 \mathrm{cou}-$ ples responded to the flyers and questionnaires were sent to them but 501 couples (84\%) sent the questionnaires back. After screening the data, 27 couples $(5.4 \%)$ were excluded from the data analysis because they either did not fill out the questionnaires properly or husbands and wives' answers were identical. The final sample includes data from 474 couples $(N=948$, for more information about demographics see Table 1). The husbands' mean age was 36.5 years $(S D=7.7)$ and the wives' mean age was 34.4 years $(S D=7.3)$. On average, couples had been married for 9.4 years $(S D=7.9)$. Eighty-four percent of the couples (398 couples) had at least one child $(M=1.0, S D=.66$, range $0-3) .{ }^{1}$ On average, husbands earned a monthly salary of 6,000 to $10,000 \mathrm{CNY}$ (approximately $\$ 1,000$ to $\$ 1,700$ USD) whereas wives earned around 2,000 to $6,000 \mathrm{CNY}$ (approximately $\$ 350$ to $\$ 1,000$ USD). ${ }^{2}$ Only $2 \%$ of the wives were unemployed and reported being housewives; all other participants worked full time. In all, the features of demographics show that our sample

\footnotetext{
${ }^{1}$ In rural areas, couples are allowed to raise more than one child.

${ }^{2}$ According to reports released by the Ministry of Human Resources and Social Security of China in 2014, the average salary varies from city to city on the basis of the economy level. The average salary in developed areas, like Beijing and Shanghai, can reach more than 4,000 to $8,000 \mathrm{CNY}$ per month (around 700 to 800 USD), while the average salary in less developed areas, like Western parts of China, just range from 1,000 to 3,000 CNY (around 160 to 500 USD). Thus, our participants' average salary falls into the category of the middle class. In addition, according to reports and the Ministry, in general, Chinese couple work full time for 8 hours per day, which can guarantee their social welfare. Husbands earn more in our sample than women. This is mainly due to the fact that men hold higher positions than wives (see Table 1).
} 
Table 1

Characteristics of the Couples

\begin{tabular}{|c|c|c|}
\hline Characteristics & Husbands: $n(\%)$ & Wives: $n(\%)$ \\
\hline \multicolumn{3}{|l|}{ Age (in years) } \\
\hline $20-29$ & $92(19 \%)$ & $157(33 \%)$ \\
\hline $30-39$ & $248(52 \%)$ & $207(44 \%)$ \\
\hline $40-49$ & $101(21 \%)$ & $95(20 \%)$ \\
\hline $50-59$ & $32(7 \%)$ & $15(3 \%)$ \\
\hline Above 59 & $1(1 \%)$ & - \\
\hline \multicolumn{3}{|l|}{ Number of children } \\
\hline 0 & $76(16 \%)$ & $76(16 \%)$ \\
\hline 1 & $339(72 \%)$ & $339(72 \%)$ \\
\hline 2 & $44(9 \%)$ & $44(9 \%)$ \\
\hline 3 & $15(3 \%)$ & $15(3 \%)$ \\
\hline \multicolumn{3}{|l|}{ Education level } \\
\hline Postgraduate & $91(19 \%)$ & $84(18 \%)$ \\
\hline Undergraduate & $191(40 \%)$ & $176(38 \%)$ \\
\hline Vocational college (3-year) & $111(24 \%)$ & $130(27 \%)$ \\
\hline Middle school or below & $81(17 \%)$ & $84(17 \%)$ \\
\hline \multicolumn{3}{|l|}{ Monthly income level (CNY) } \\
\hline $900-2,000$ & $45(9 \%)$ & $85(8 \%)$ \\
\hline $2,001-6,000$ & $210(44 \%)$ & $222(47 \%)$ \\
\hline $6,001-10,000$ & $138(29 \%)$ & $118(25 \%)$ \\
\hline $10,001-15,000$ & $61(13 \%)$ & $33(4 \%)$ \\
\hline Above 15,000 & $20(4 \%)$ & $8(2 \%)$ \\
\hline No salary & - & $8(2 \%)$ \\
\hline \multicolumn{3}{|l|}{ Occupation } \\
\hline Civil servants and public institutions & $163(35 \%)$ & $180(38 \%)$ \\
\hline State-owned enterprises & $66(14 \%)$ & $66(14 \%)$ \\
\hline Private or foreign companies or factories & $158(33 \%)$ & $170(36 \%)$ \\
\hline Self-employed, entrepreneurship, and others & $85(17 \%)$ & $47(10 \%)$ \\
\hline Retired & $2(1 \%)$ & $4(1 \%)$ \\
\hline Unemployed & - & $7(2 \%)$ \\
\hline \multicolumn{3}{|l|}{ Occupational status } \\
\hline Lower white-collar & $309(65 \%)$ & $367(78 \%)$ \\
\hline Middle white-collar & $132(28 \%)$ & $86(18 \%)$ \\
\hline Higher positions (e.g., manger, chairperson) & $33(7 \%)$ & $14(3 \%)$ \\
\hline Unemployed & - & $7(2 \%)$ \\
\hline \multicolumn{3}{|l|}{ Residence } \\
\hline Urban areas (cities and counties) & $386(81 \%)$ & $386(81 \%)$ \\
\hline Rural areas (villages) & $88(19 \%)$ & $88(19 \%)$ \\
\hline \multicolumn{3}{|l|}{ Marital duration } \\
\hline $1-9$ & $262(60 \%)$ & $260(60 \%)$ \\
\hline $10-19$ & $109(22 \%)$ & $112(24 \%)$ \\
\hline $20-29$ & $62(13 \%)$ & $62(13 \%)$ \\
\hline 30-39 & $14(3 \%)$ & $13(3 \%)$ \\
\hline
\end{tabular}

was similar to the Chinese population in terms of income, job type, and number of children but our sample had a better education level than the nation average. ${ }^{3}$ All the procedures are primarily approved by the researchers' university Institutional Review Board.

\section{Measures}

Demographics. Participants reported their age, marital duration, education, monthly in- come, number of children, job type, job position, and area of residence.

External stress and internal stress. In order to assess external and internal stress, we translated the English version of the Multidimensional Stress Questionnaire for couples into

\footnotetext{
${ }^{3}$ According to the government report about the sixth national population census, the average education level of the Chinese population is middle school.
} 
Chinese (MSQ-C; Bodenmann et al., 2007). The MSQ-C assesses external stress with eight items and internal stress with 10 items. Participants report first about external stress (e.g., work stress, financial problems, social contacts with colleagues and friends, parenting) and then respond to questions about experiences of internal stress (e.g., differences of opinion with your partner, unsatisfactory distribution of duties and responsibilities) over the past 12 months. An aggregated score (either eight items for external stress or 10 items for internal stress) represents an individual's external or internal stress level respectively. All the scales are rated on a 4-point Likert scale $(1=$ not at all stressful to $4=$ very stressful). Two bilinguals (English and Chinese) first translated the published English version of MSQ-C to Chinese and then backtranslated it to English to edit an accurate Chinese version. Internal consistency was acceptable for external stress (.69 for wives and .69 for husbands) and good for internal stress (.75 for wives and .80 for husbands).

Dyadic coping. We used the validated Chinese version of the Dyadic Coping Inventory (DCI; Xu, Hilpert, Randall, Li, \& Bodenmann, 2016) to assess dyadic coping behaviors. Couples rated how often they (self) and their partners (partner) engaged in stress communication and dyadic coping (DC) strategies on a 5-point Likert scale $(1=$ not at all/very rarely to $5=$ very often). The validated Chinese version of DCI contains five subscales (stress communication, supportive DC, delegated DC, negative DC and common DC), with two additional evaluation items for measuring the efficiency of using DC skills. For the present study, we assessed Chinese couples' perception of the usage of three positive forms of dyadic coping skills (Bodenmann, 2005, 2008) through aggregating the following three subscales with a total of 12 items: supportive dyadic coping by partner (where one partner voluntarily helps the stressed spouse: e.g., "My partner helps me to see the stressful situations in a different light"); delegated dyadic coping partner (where one partner is explicitly asked to provide support: e.g., "I take on things that my partner would normally do in order to help him/her out"), and common dyadic coping (where both partners engage in communal problem-solving activities: e.g., "We try to cope with the problem together and search for ascertained solutions").
A meta-analysis of studies concerning the association between dyadic coping and relationship satisfaction provides strong evidence that all three forms of positive dyadic coping strongly predict Western couples' relationship satisfaction (Falconier et al., 2015). The internal consistency of the aggregated scale in the current study was $\alpha=.90$ for wives as well as for husbands.

Marital satisfaction. To assess relationship satisfaction, we made use of the validated Chinese version (Wang et al., 2012) of the Relationship Assessment Scale (RAS; Hendrick, Dicke, \& Hendrick, 1998). The seven-item RAS is rated on a 5-point scale asking spouses to rate the extent to which they are satisfied or dissatisfied with their marital situation (e.g., "In general, how satisfied are you with your relationship?"). In the present study, the internal consistency is $\alpha=.90$ for wives and $\alpha=.88$ for husbands.

\section{Statistical Analysis}

We used an actor-partner interdependence mediation model (APIMeM; Ledermann, Macho, \& Kenny, 2011) to test the hypotheses. The APIMeM approach allows us to model the interdependence between husbands' and wives' factors through specifying both actor and partner effects as well as simultaneously including mediators of internal stress and dyadic coping (see Figure 1). The model was developed in a stepwise procedure. First, we specified a saturated model with all variables, including a variety of control variables such as marital duration, area of residence, education level, income level, job type and positions as well as number of children. In order to simplify the model, control variables would be removed from the model if they did not significantly predict any variable. Second, we explored gender differences through comparing the saturated model, where paths were allowed to vary freely, with a model where actor and partner effects were constrained to be equal across gender. Given that the constrained model did not worsen the model fit relative to the unconstrained model using a chi square difference test, we maintained the more parsimonious model with invariant effects across gender. In addition, a complete mediation effect would be identified if the total indirect effect between the one predic- 


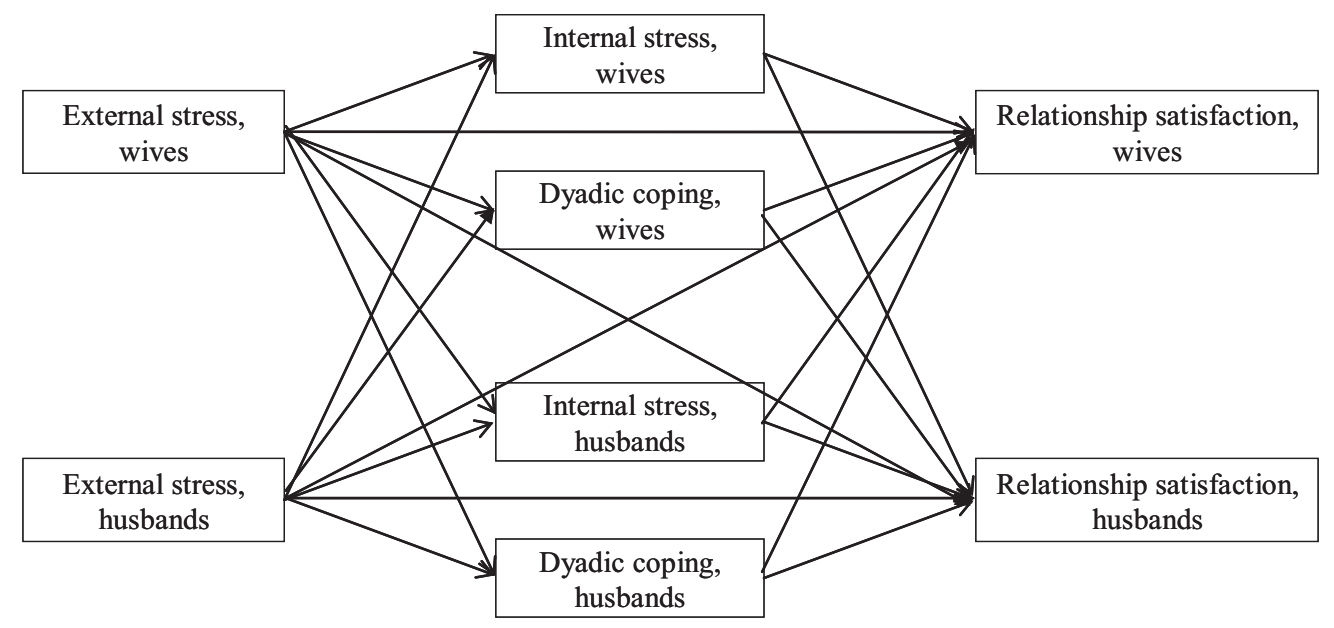

Figure 1. The conceptual model. The actor-partner interdependence mediation model (APIMeM) conceptualizes Chinese couples' internal stress and dyadic coping as mediators between external stress and relationship satisfaction. Control variables were tested by adding them as exogenous variables with all study variables. Correlations and residual covariances between the study variables were calculated but are uncharted.

tor and one outcome (e.g., external stress and dyadic coping) is nonzero and the direct effect between the same predictor and outcome (e.g., external stress and relationship satisfaction) is zero (Ledermann et al., 2011).

However, it is crucial to note that there are several mediation paths (see Figure 1), but we only tested the conceptually meaningful pathways. The meaningful paths involve (a) own external stress; (b) perceived own and the partner's dyadic coping or internal stress as a response to own external stress; and (c) own relationship satisfaction. These indirect paths are conceptually meaningful because they are linked by the inherent dynamics (i.e., my external stress can intensify my own and my partner's perception of internal stress and anticipate my own or my partner's dyadic coping behaviors). There are other indirect paths, but they are less interpretable because they involve different situations (e.g., the effect of the linkage between one partner's external stress and my own internal stress or dyadic coping to predict my own relationship satisfaction). Thus, the current study tested those indirect paths which are conceptually and empirically contributive to understand stress spillover effects on Chinese couples.

The statistical software package $\mathrm{R}$ ( $\mathrm{R}$ version 3.1, R Core Team, 2008) was used to compute descriptive statistics. To compute the APIMeM, we used the lavaan package 0.5-16 (Rosseel,
2012) in R. We relied on common fit indices to test the model fit of the data: chi square $\left(\chi^{2}\right)$, root mean square error of approximation (RMSEA), comparative fit index (CFI) and standardized root mean squared residual (SRMR). A good model to data fit is indicated by CFI above .95, RMSEA smaller than .05, and SRMR smaller than.08 (Marsh, Hau, \& Wen, 2004). We used full information maximum likelihood to deal with missing data. As in general one cannot assume a normal distribution of all the indirect effects, we reported the bootstrapped (5,000 times) standard errors (Preacher \& Hayes, 2008).

\section{Results}

\section{Descriptive Analysis}

Table 2 presents means, standard deviations, and intercorrelations for all study variables. Wives reported significantly higher scores of internal stress $(p=.01)$, indicating that wives may perceive more internal stress than their partners. Furthermore, husbands reported significantly higher scores of dyadic coping in comparison to their wives $(p=.01)$. Although these mean differences were significant, effect sizes were small $\left(d_{\text {internal stress }}=.05 ; d_{\text {dyadic }}\right.$ coping $=.13$ ). Furthermore, all variables were correlated in the expected directions: external stress and relationship satisfaction $\left(r_{\text {Wives }}=\right.$ 
Table 2

Descriptive Statistics and Correlations Among All Studied Variables

\begin{tabular}{|c|c|c|c|c|c|c|c|c|c|c|}
\hline \multirow[b]{3}{*}{ Variables } & \multicolumn{6}{|c|}{ Descriptive statistics } & \multirow{2}{*}{\multicolumn{4}{|c|}{$\begin{array}{c}\text { Correlations } \\
\text { Variables }\end{array}$}} \\
\hline & \multicolumn{2}{|c|}{ Wives } & \multicolumn{2}{|c|}{ Husbands } & \multirow[b]{2}{*}{$p$} & \multirow[b]{2}{*}{$d$} & & & & \\
\hline & $M$ & $S D$ & $M$ & $S D$ & & & 1 & 2 & 3 & 4 \\
\hline 1. External stress & 2.39 & .49 & 2.35 & .48 & .07 & .08 & .38 & .55 & -.16 & -.22 \\
\hline 2. Internal stress & 2.39 & .47 & 2.32 & .50 & .01 & .12 & .59 & .37 & -.30 & -.33 \\
\hline 3. Dyadic coping & 3.46 & .45 & 3.52 & .46 & .01 & .13 & -.12 & -.28 & .36 & .37 \\
\hline 4. Relationship satisfaction & 3.42 & .50 & 3.47 & .51 & .12 & .05 & -.22 & -.31 & .39 & .38 \\
\hline
\end{tabular}

Note. Correlations above the diagonal are for husbands and below the diagonal are for wives. Correlations between husbands and wives are shown between the diagonal. $d=$ Cohen's d. Significant correlations are in bold ( $p<.05$; two-tailed).

$\left.-.22 ; r_{\text {Husbands }}=-.16\right)$; internal stress and relationship satisfaction $\left(r_{\text {Wives }}=-.31 ; r_{\mathrm{Hus}^{-}}\right.$ bands $=-.33)$; dyadic coping and relationship satisfaction $\left(r_{\text {Wives }}=.39 ; r_{\text {Husbands }}=.37\right)$.

\section{Main Results}

We first tested two conceptual models with and without all control variables. None of the control variables had any significant effect and were therefore dropped from the two competing models. The chi-square difference test shows that the model with constraining the paths to be equal across gender was not worse than the model allowing all paths to be freely estimated, $\chi^{2}(9)=9.37, p=.41$. Thus, the constrained model fitted the data well $\left(\chi^{2}(10)=15.16, p=\right.$ .13 , CFI $=.99$, RMSEA $=.03$, CI $[.00,06]$, SRMR $=.03$ ).

Direct effects. Table 3 shows all the results. As the constrained model fitted the data well, we conclude that there were no gender differences in stress and dyadic coping processes in Chinese couples. In more detail, although we found mean level differences in internal stress and dyadic coping across gender, no gender differences were found when examining how these variables were associated with each other. Second, direct paths from both gender' external stress to relationship satisfaction were not statistically significant $\left(\beta_{\text {actor }}=.01\right.$, $\left.p=.85 ; \beta_{\text {partner }}=-.05, p=.12\right)$. Associations between external stress and internal stress for both actor and partner effects were significant $\left(\beta_{\text {actor }}=.12, p=.00 ; \beta_{\text {partner }}=.52, p=.00\right)$. Moreover, internal stress negatively predicted relationship satisfaction $\left(\beta_{\text {actor }}=-.15, p=\right.$ $\left..00 ; \beta_{\text {partner }}=-.09, p=.01\right)$, whose negative impact was confirmed. Third, dyadic coping behaviors were negatively associated with external stress in both actor and partner effects $\left(\beta_{\text {actor }}=-.08, p<.03 ; \beta_{\text {partner }}=-.15, p<\right.$ $.00)$. However, positive associations between dyadic coping and relationship satisfaction were only established in actor effects for both gender $(\beta=.25, p<.00)$ instead of in partner effects for both gender $(\beta=.04, p<.19)$, suggesting that only spouses' positive dyadic coping behaviors (e.g., supportive DC or common DC behaviors) can improve their own relationship satisfaction. $^{4}$

Indirect effects. In accordance with our main hypotheses, we assumed that Chinese couples' internal stress and dyadic coping would mediate the association between external stress and relationship satisfaction through two conceptually interpretable pathways: the actoractor effect and the partner-partner effect. The actor-actor effect indicates that both wives' and husbands' own external stress can decrease or improve their sense of relationship satisfaction via their own perception of internal stress and dyadic coping. Results support the assumption of the actor-actor effect for both genders $\left(\beta_{\text {internal stress }}=-.08, p<.00 ; \beta_{\text {dyadic coping }}=\right.$ $-.04, p<.00)$. Thus, the more both partners employed positive dyadic coping skills, the more they would be satisfied with their relation-

\footnotetext{
${ }^{4}$ We tested a post hoc model if the stress-coping processes might be different for couples with children $(n=$ 398) and without children $(n=76)$ in a multigroup APIMeM model. The model constraining all paths to be equal between couples with and without children fitted the data well, showing that there are no group differences. Therefore, we included all couples in the final model.
} 
Table 3

Direct Effects and Indirect Effects for the APIMeM

\begin{tabular}{|c|c|c|c|c|c|}
\hline Effects & Estimate & $S E$ & $z$ & $p$ & CI $95 \%$ bias-corrected \\
\hline \multicolumn{6}{|l|}{ Direct effects } \\
\hline \multicolumn{6}{|l|}{ Actor effects } \\
\hline $\mathrm{ES} \rightarrow \mathrm{RS}$ & .01 & .04 & .19 & .85 & {$[-.07, .08]$} \\
\hline $\mathrm{IS} \rightarrow \mathrm{RS}$ & -.15 & .04 & -3.77 & .00 & {$[-.22,-.07]$} \\
\hline $\mathrm{DC} \rightarrow \mathrm{RS}$ & .25 & .04 & 7.17 & .00 & {$[.18, .32]$} \\
\hline $\mathrm{ES} \rightarrow \mathrm{IS}$ & .12 & .03 & 3.78 & .00 & {$[.06, .18]$} \\
\hline $\mathrm{ES} \rightarrow \mathrm{DC}$ & -.08 & .04 & -2.17 & .03 & {$[-.15,-.00]$} \\
\hline \multicolumn{6}{|l|}{ Partner effects } \\
\hline $\mathrm{ES} \rightarrow \mathrm{RS}$ & -.05 & .04 & -1.54 & .12 & {$[-.12, .02]$} \\
\hline $\mathrm{IS} \rightarrow \mathrm{RS}$ & -.09 & .04 & -2.50 & .01 & {$[-.16,-.02]$} \\
\hline $\mathrm{DC} \rightarrow \mathrm{RS}$ & .04 & .03 & 1.33 & .19 & {$[-.02, .10]$} \\
\hline $\mathrm{ES} \rightarrow \mathrm{IS}$ & .52 & .03 & 15.71 & .00 & {$[.46, .59]$} \\
\hline $\mathrm{ES} \rightarrow \mathrm{DC}$ & -.15 & .04 & -3.65 & .00 & {$[-.23,-.07]$} \\
\hline \multicolumn{6}{|l|}{ Indirect effects } \\
\hline \multicolumn{6}{|l|}{ Actor-actor effects } \\
\hline $\mathrm{ES} \rightarrow \mathrm{IS} \rightarrow \mathrm{RS}$ & -.08 & .02 & -3.67 & .00 & {$[-.12,-.03]$} \\
\hline $\mathrm{ES} \rightarrow \mathrm{DC} \rightarrow \mathrm{RS}$ & -.04 & .01 & -3.03 & .00 & {$[-.06,-.01]$} \\
\hline \multicolumn{6}{|l|}{ Partner-partner effects } \\
\hline $\mathrm{ES} \rightarrow \mathrm{IS} \rightarrow \mathrm{RS}$ & -.01 & .01 & -1.43 & .15 & {$[-.03, .00]$} \\
\hline $\mathrm{ES} \rightarrow \mathrm{DC} \rightarrow \mathrm{RS}$ & -.03 & .02 & -1.36 & .26 & {$[.00, .01]$} \\
\hline
\end{tabular}

Note. $\quad$ APIMeM = actor-partner interdependence mediation model; $S E=$ standard error; $\mathrm{ES}=$ external stress; IS = internal stress; RS = relationship satisfaction; $\mathrm{DC}=$ dyadic coping; CI = confidence interval. As final results (standardized analyses) reveal no gender differences, regression weights are identical for husbands and wives and are reported only one time. Significant coefficients are in bold ( $p<.05$; two-tailed).

ship. In contrast, the partner-partner effect indicates that the negative effect of wives' own external stress on relationship satisfaction can be potentially influenced or alleviated via their partners' internal stress and dyadic coping. Results did not support the proposed effect for either gender $\left(\beta_{\text {internal stress }}=-.01, p=.15\right.$; $\left.\beta_{\text {dyadic coping }}=-.03, p=.26\right)$. One explanation is that direct partner effects of dyadic coping on relationship satisfaction were not significant.

Because the magnitude of the mediation effect of internal stress appeared to be stronger than that of the mediation effect dyadic coping, we compared these two mediation effects by constraining the indirect paths to be equal in a post hoc model test. The chi-square difference test shows that internal stress is a stronger mediator for the stress spillover effects, $\chi^{2}(11)=$ $16.27, p=.01$.

\section{Discussion}

According to the STM, internal stress and dyadic coping function as explaining mediators for the link between external stress and relation- ship satisfaction. However, family researchers need to explore these effects beyond Western samples to consolidate the established model and meanwhile test for gender differences in these effects. To address this gap, utilizing a sample of community-dwelling Chinese couples, the current study sought to examine stress and dyadic coping processes in an Eastern culture.

The effect sizes (see Table 2) show that gender differences in how Chinese couples perceived the stress-coping processes were subtle. This indicates that both men and women may experience similar levels of stress and perceive similar levels of partner's behavior, which in turn explains the fact that both partners have a relatively similar level of relationship satisfaction. Hardly establishing gender differences in mean level differences of the study variables provides initial evidence for our assumption that the couples of our age group have gone through the transition from traditional cultures to contemporary values. Thus, couples may perceive the same amount of stress and dyadic coping in a similar way. 
In relation to direct effects, all direct pathways were significant with two exceptions (see Table 3). First, although external stress and relationship satisfaction correlated significantly with each other, this association was no longer significant when we included the mediator variables in the model, indicating that the mediators can fully explain the association between external stress and relationship satisfaction. Second, we did not find significant partner effects between dyadic coping and relationship satisfaction, replicating a previous study with Western couples (Hilpert et al., 2013). In addition, findings show that the associations between the variables are equal across gender, further supporting the assumption that Chinese husbands and wives may perceive similar levels of stress, coping behaviors, and relationship satisfaction. This means that the effect of external stress on one's own behavior is very similar between partners when reacting to stressors and perceiving partners' coping behaviors. We can conclude that under everyday stressful circumstances, modern Chinese couples demand coping resources from their partners to maintain their own psychological well-being.

In the subsequent steps, we tested if internal stress and dyadic coping could mediate the association between external stress and relationship satisfaction and the results support our hypotheses. These findings are in line with prior studies reporting that internal stress is a strong mediator in Western couples (e.g., Bodenmann et al., 2007; Ledermann et al., 2010). As for Chinese couples, external stress would have a deleterious effect on their levels of relationship satisfaction through activating their perceptions of internal stress in a similar pattern. These findings also support our assumption that modern dual-earner couples reside in stressful environments where both are working full time and stress can hinder their adaptive relationship. Further, we assume that the lack of gender differences may be also due to the severity of modern stressors, which might motivate them to ask for support. Several lines of studies report that Chinese couples consistently suffer several stressors in modern China, such as the soaring costs of accommodation (Chen, Hao, \& Stephens, 2010) and the intergenerational stress of supporting their parents and children caused by the one-child policy (Hesketh et al., 2005; Shek, 2006). Should it be the case, both partners might be intolerant of stressors and demand coping resources from each other to cope with stress.

Dyadic coping can mediate the association between external stress and relationship satisfaction as well, which is in line with studies with Western couples (e.g., Hilpert et al., 2015; Donato et al., 2015). We want to further highlight that all mediators were entered in the model simultaneously, which means that the effect of a specific mediator is controlled for the effect of the other mediator. In order to reduce the negative effect of stress, Chinese couples help each other to adapt to stressful situations by providing support to each other. Additionally, the lack of gender differences was attributed to the influences of contemporary values that play a pivotal role in shaping Chinese couples' stress reactivity and coping behaviors.

Although we found that internal stress and dyadic coping were mediators when testing actor-actor effects (own stress-perceived partner's behavior-own relationship satisfaction), yet we did not find the same pattern for partnerpartner effects. This is conceptually sound as these mediation paths are not connected to individuals' own stressful experiences. Partner B's coping behaviors and sense of relationship satisfaction would be hardly influenced by Partner A' stressful experiences, especially when both partners are stressed. This highlights that the actor-actor mediation effects are conceptually validated mechanisms and these finding are consistent with studies with Western couples (e.g., Wunderer \& Schneewind, 2008).

Finally, as we found that the magnitudes of the two mediation paths were different, we compared them in a post hoc test. Results show that internal stress is a stronger mediator than dyadic coping. This is surprising because maintaining relationship harmony is still highly valued for Chinese couples (Xu \& Hiew, 2016). This finding is also contrary to findings with Western couples. For instance, Hilpert and colleagues (2013) reported that the mediation effect of dyadic coping was significantly stronger than couples' conflict behaviors. Thus, this finding might indicate that Chinese couples undergo higher levels of stressor that might exacerbate more internal stress (e.g., conflict interactions) between partners. Future work should examine whether dyadic coping can reduce the internal stress level. 


\section{Limitations}

Several limitations have to be mentioned. First, our sample mainly consisted of couples with relatively younger children. Therefore, results cannot be generalized to newlyweds or couples with older children. Second, couples who were born in the 1970s tend to be more traditional or collectivistic than couples born in the 1980s and 1990s, who tend to be more individualistic (Shu \& Zhu, 2009). In order to account for this variability, we controlled for several important aspects (e.g., age, education, marital duration, number of children) to make the results more comparable. However, future studies should collect data from a more diverse generation group to examine stress and dyadic coping processes. Finally, it is not possible to draw causal conclusions about the underlying mechanisms based on our crosssectional data. Future research should focus on designing longitudinal (e.g., diary study) or experimental studies to deepen our understanding of stress and dyadic coping mechanisms in Chinese couples.

\section{Theoretical and Practical Implications}

This study has theoretical implications for future research. For the first time we provided evidence that the STM, originated in Western couples, can be applied to couples in a typical Eastern culture. The results support the theoretical conceptualization of stress spillover and relevant dyadic coping behaviors beyond Western cultures (Bodenmann, 2005). Given the application in Chinese couples, future studies should facilitate the STM to be applied to more cultural samples to consolidate our findings or provide other novel findings.

Furthermore, the current study indicates some practical implications for researchers and clinicians who work with Chinese couples. First, in terms of the cultural effects, practitioners should try to make couples be fully aware of the cultural doctrines that are constantly influenced by modern society. As a consequence, Chinese couples may find the balance between traditional and modern cultural values, which might be a stress for the partners. To keep the balance between gender hierarchy and equitable responsibilities assigned to men and women is extremely beneficial for main- taining their relationship satisfaction and well-being. In addition, adjusting traditional and modern values between partners is also quite important to maintain harmony in the long run. Second, Chinese couples can be very sensitive to both external and internal stressors. Couple clinicians should take this fact into considerations when providing counseling to Chinese couples. Meanwhile, clinicians should attach more importance to couples' perception of internal stress as it seems to be more deleterious for their relationships. Clinicians can emphasize that couples should engage in dyadic coping to reduce the stress level. This thought is extremely meaningful and beneficial for Chinese couples who live in a collectivistic culture. Future research can implement relationship education programs to Chinese couples (see Halford \& Bodenmann, 2013) to improve their conflict resolution and dyadic coping skills. However, these trainings need rigorous clinical studies and cultural adaptations (e.g., communication style, mode of providing support, and factors of caring for children) before we can certify that they are functional and beneficial in the fast-changing environment in China.

\section{Conclusion}

In sum, we confirmed mediating effects of internal stress and dyadic coping in the association between external stress and relationship satisfaction and tested gender differences in these effects beyond Western cultures. Chinese couples who live in a collectivistic culture can regard dyadic coping behaviors as potential resources to cope with stress. In this way, for researchers and clinicians, the current study can be culturally illuminative for future investigations of stress and coping mechanisms in diverse groups and serves as a reference for designing culturally diverse therapy and prevention programs for couples.

\section{References}

Bodenmann, G. (2005). Dyadic coping and its significance for marital functioning. In T. A. Revenson, K. Kayser, \& G. Bodenmann (Eds.), Couples coping with stress: Emerging perspectives on $d y$ adic coping (pp. 35-50). Washington, DC: American Psychological Association. http://dx.doi.org/ 10.1037/11031-002 
Bodenmann, G. (2008). Dyadisches Coping Inventar (DCI). Test-manual [Dyadic Coping Inventory (DCI). Test manual]. Bern, Switzerland: Huber.

Bodenmann, G., Ledermann, T., \& Bradbury, T. N. (2007). Stress, sex, and satisfaction in marriage. Personal Relationships, 14, 551-569. http://dx.doi .org/10.1111/j.1475-6811.2007.00171.x

Bodenmann, G., Meuwly, N., Germann, J., Nussbeck, F. W., Heinrichs, M., \& Bradbury, T. N. (2015). Effects of stress on the social support provided by men and women in intimate relationships. Psychological Science, 26, 1584-1594. http://dx .doi.org/10.1177/0956797615594616

Buck, A. A., \& Neff, L. A. (2012). Stress spillover in early marriage: The role of self-regulatory depletion. Journal of Family Psychology, 26, 698-708. http://dx.doi.org/10.1037/a0029260

Chen, F. M., \& Li, T. S. (2007). Marital enqing: An examination of its relationship to spousal contributions, sacrifices, and family stress in Chinese marriages. The Journal of Social Psychology, 147, 393-412. http://dx.doi.org/10.3200/SOCP.147.4 393-412

Chen, J., Hao, Q., \& Stephens, M. (2010). Assessing housing affordability for post-reform China: A case study of Shanghai. Housing Studies, 25, $877-$ 901. http://dx.doi.org/10.1080/02673037.2010 .511153

Donato, S., Parise, M., Iafrate, R., Bertoni, A., Finkenauer, C., \& Bodenmann, G. (2015). Dyadic coping responses and partners' perceptions for couple satisfaction: An actor-partner interdependence analysis. Journal of Social and Personal Relationships, 32, 580-600. http://dx.doi.org/10.1177/ 0265407514541071

Ebrey, P. (2006). Confucianism. In D. S. Browning, M. C. Green, \& J. W. Jr (Eds.), Sex, marriage, and family in world religions (pp. 367-450). New York, NY: Columbia University Press.

Falconier, M. K., Jackson, J. B., Hilpert, P., \& Bodenmann, G. (2015). Dyadic coping and relationship satisfaction: A meta-analysis. Clinical Psychology Review, 42, 28-46. http://dx.doi.org/ 10.1016/j.cpr.2015.07.002

Halford, W. K., \& Bodenmann, G. (2013). Effects of relationship education on maintenance of couple relationship satisfaction. Clinical Psychology Review, 33, 512-525. http://dx.doi.org/10.1016/j.cpr .2013.02.001

Hendrick, S. S., Dicke, A., \& Hendrick, C. (1998). The Relationship Assessment Scale. Journal of Social and Personal Relationships, 15, 137-142. http://dx.doi.org/10.1177/0265407598151009

Herzberg, P. Y. (2013). Coping in relationships: The interplay between individual and dyadic coping and their effects on relationship satisfaction. Anxiety, Stress, \& Coping, 26, 136-153. http://dx.doi .org/10.1080/10615806.2012.655726
Hesketh, T., Lu, L., \& Xing, Z. W. (2005). The effect of China's one-child family policy after 25 years. The New England Journal of Medicine, 353, 11711176. http://dx.doi.org/10.1056/NEJMhpr051833

Hilpert, P., Bodenmann, G., Nussbeck, F. W., \& Bradbury, N. T. (2013). Predicting relationship satisfaction in distressed and non-distressed couples based on a stratified sample: A matter of conflict, positivity, or support? Family Science, 4, 110-120. http://dx.doi.org/10.1080/19424620 .2013 .830633

Hilpert, P., Kuhn, R., Anderegg, V., \& Bodenmann, G. (2015). Comparing simultaneously the effects of extra-dyadic and intra-dyadic experiences on relationship outcomes. Family Science, 6, 129142. http://dx.doi.org/10.1080/19424620.2015 .1082018

Ledermann, T., Bodenmann, G., Rudaz, M., \& Bradbury, T. N. (2010). Stress, communication, and marital Quality, Family Relations, 59, 195206. http://dx.doi.org/10.1111/j.1741-3729.2010 $.00595 . \mathrm{x}$

Ledermann, T., Macho, S., \& Kenny, D. A. (2011). Assessing mediation in dyadic data using actorpartner interdependence model. Structural Equation Modeling, 18, 595-612. http://dx.doi.org/10 $.1080 / 10705511.2011 .607099$

Ling, Y., \& Powell, G. N. (2001). Work-family conflict in contemporary China: Beyond an American-based model. International Journal of Cross Cultural Management, 1, 357-373. http://dx.doi .org/10.1177/147059580113006

Marsh, H. W., Hau, K. T., \& Wen, Z. (2004). In search of golden rules: Comment on hypothesistesting approaches to setting cutoff values for fit indexes and dangers in overgeneralizing $\mathrm{Hu}$ and Bentler's (1999) findings. Structural Equation Modeling, 11, 320-341. http://dx.doi.org/10.1207/ s15328007sem1103_2

Marshall, T. C. (2008). Cultural differences in intimacy: The influence of gender-role ideology and individualism-collectivism. Journal of Social and Personal Relationships, 25, 143-168. http://dx.doi .org/10.1177/0265407507086810

Oyserman, D., Coon, H. M., \& Kemmelmeier, M. (2002). Rethinking individualism and collectivism: Evaluation of theoretical assumptions and meta-analyses. Psychological Bulletin, 128, 3-72. http://dx.doi.org/10.1037/0033-2909.128.1.3

Preacher, K. J., \& Hayes, A. F. (2008). Asymptotic and resampling strategies for assessing and comparing indirect effects in multiple mediator models. Behavior Research Methods, 40, 879-891. http://dx.doi.org/10.3758/BRM.40.3.879

Quek, K. M., Knudson-Martin, C., Rue, D., \& Alabiso, C. (2010). Relational harmony: A new model of collectivism and gender equality among Chinese American couples. Journal of 
Family Issues, 31, 358-380. http://dx.doi.org/10 .1177/0192513X09351162

R, Development Core Team. (2008). R: A language and environment for statistical computing (Version 3.1) [Computer software].Vienna, Austria: R Foundation for Statistical Computing. Retrieved from http://www.R-project.org

Randall, A. K., \& Bodenmann, G. (2009). The role of stress on close relationships and marital satisfaction. Clinical Psychology Review, 29, 105-115. http://dx.doi.org/10.1016/j.cpr.2008.10.004

Repetti, R. L. (1989). Effects of daily workload on subsequent behavior during marital interaction: The roles of social withdrawal and spouse support. Journal of Personality and Social Psychology, 57, 651-659. http://dx.doi.org/10.1037/0022-3514.57 .4 .651

Revenson, T. A., \& Lepore, S. J. (2012). Stress and coping processes in social context. In A. Baum, T. A. Revenson, \& J. E. Singer (Eds), Handbook of health psychology (pp. 193-217). New York, NY: Psychology Press.

Rosseel, Y. (2012). Lavaan: An R package for structural equation modeling. Journal of Statistical Software, 48, 1-36. http://dx.doi.org/10.18637/jss .v048.i02

Shek, D. T. L. (2006). Chinese family research: Puzzles, paradigms, and policy implications. Journal of Family Issues, 27, 275-284. http://dx.doi.org/10 $.1177 / 0192513 X 05283508$

Shu, X., \& Zhu, Y. (2009). The quality of life in China. Social Indicators Research, 92, 191-225. http://dx.doi.org/10.1007/s11205-008-9350-4

Wang, X. Y., Xiao, J., Cui, L. X., Jin, X. Y., He, L. K., \& Han, X. (2012). The correlations of uni- versity students love style, dysfunctional attitude and love satisfaction and life satisfaction. Chinese Journal of Behavioral Medical Science, 6, 555557.

Wunderer, E., \& Schneewind, K. A. (2008). The relationship between marital standards, dyadic coping and marital satisfaction. European Journal of Social Psychology, 38, 462-476. http://dx.doi .org/10.1002/ejsp.405

Xu, F., \& Hiew, D. N. (2016). Dyadic coping in Chinese couples. In M. K. Falconier, A. K. Randall, \& G. Bodenmann (Eds.), Couples coping with stress: A cross-cultural perspective (pp. 218-235). New York, NY: Routledge.

Xu, F., Hilpert, P., Randall, A. K., Li, Q., \& Bodenmann, G. (2016). Validation of the Dyadic Coping Inventory with Chinese couples: Factorial structure, measurement invariance, and construct validity. Psychological Assessment, 28, e127-e140. http://dx.doi.org/10.1037/pas0000329

Zhang, M., Foley, S., \& Yang, B. Y. (2013). Workfamily conflict among Chinese married couples: Testing spillover and crossover effects. The International Journal of Human Resource Management, 24, 3213-3231. http://dx.doi.org/10.1080/ 09585192.2013.763849

Zuo, J. (2003). From revolutionary comrades to gendered partners: Marital construction of breadwinning in post-Mao urban China. Journal of Family Issues, 24, 314-337. http://dx.doi.org/10.1177/ 0192513X02250888 